\title{
Percepções de enfermeiros sobre o cuidado às mulheres com diagnóstico de câncer de
}

\section{mama}

Nurses perceptions of care for women with breast cancer diagnosis

Percepciones de las enfermeras sobre el cuidado de las mujeres com diagnóstico de cáncer de seno

Recebido: 13/01/2021 | Revisado: 21/01/2021 | Aceito: 26/01/2021 | Publicado: 02/02/2021

\author{
Camila Amthauer \\ ORCID: https://orcid.org/0000-0002-7530-9809 \\ Universidade do Oeste de Santa Catarina, Brasil \\ E-mail: camila.amthauer@hotmail.com \\ Deisi Graczyk \\ ORCID: https://orcid.org/0000-0001-7801-243X \\ Universidade do Oeste de Santa Catarina, Brasil \\ E-mail: deisy_graczyk@hotmail.com
}

\begin{abstract}
Resumo
Objetivo: conhecer a percepção de enfermeiros sobre o cuidado e as práticas desenvolvidas junto às mulheres com diagnóstico de câncer de mama. Metodologia: trata-se de uma pesquisa qualitativa, do tipo exploratória-descritiva, desenvolvida com enfermeiros que atuam no cuidado às mulheres com câncer de mama. A coleta de dados aconteceu por meio de entrevista semiestruturada, de caráter individual, respeitando a privacidade dos participantes. Os dados foram analisados por meio da análise de conteúdo do tipo temática, proposta por Minayo. Foram respeitados os princípios éticos em saúde, conforme a Resolução ${ }^{\circ}$ 466/2012. Resultados: a partir da análise, emergiram dois temas: cuidados de Enfermagem às mulheres com câncer de mama: práticas e enfrentamentos; e, dificuldades enfrentadas pelas mulheres com diagnóstico de câncer de mama, na voz dos enfermeiros. Considerações finais: percebe-se a importância de suscitar a reflexão dos enfermeiros no que concerne aos cuidados às mulheres com câncer de mama, a fim de (re)pensar a assistência ofertada em toda a dimensão biopsicosocioespiritual da mulher.
\end{abstract}

Palavras-chave: Neoplasias da mama; Cuidados de enfermagem; Enfermagem oncológica; Integralidade em saúde; Pesquisa qualitativa.

\begin{abstract}
Objective: to know the perception of nurses about the care and practices developed with women diagnosed with breast cancer. Methodology: this is a qualitative research, exploratory-descriptive, developed with nurses who work in the care of women with breast cancer. Data collection took place through semi-structured interviews, of an individual character, respecting the privacy of the participants. The data were analyzed through thematic content analysis proposed by Minayo. Ethical health principles were respected, in accordance with Resolution No. 466/2012. Results: from the analysis, two themes emerged: Nursing care for women with breast cancer: practices and confrontations; and, difficulties faced by women diagnosed with breast cancer, in the nurses' voice. Final considerations: the importance of encouraging nurses to reflect on the care of women with breast cancer is perceived, in order to (re) think the assistance offered in the entire biopsychosocio-spiritual dimension of women.
\end{abstract}

Keywords: Breast neoplasms; Nursing care; Oncology nursing; Integrality in health; Qualitative research.

\section{Resumen}

Objetivo: conocer la percepción de las enfermeras sobre los cuidados y prácticas que desarrollan con las mujeres diagnosticadas con cáncer de mama. Metodología: se trata de una investigación cualitativa, exploratoria-descriptiva, desarrollada con enfermeras que laboran en el cuidado de mujeres con cáncer de mama. La recogida de datos se realizó mediante entrevistas semiestructuradas, de carácter individual, respetando la privacidad de los participantes. Los datos fueron analizados mediante el análisis de contenido temático propuesto por Minayo. Se respetaron los principios éticos de salud, de acuerdo con la Resolución No. 466/2012. Resultados: del análisis surgieron dos temas: Atención de enfermería a mujeres con cáncer de mama: prácticas y enfrentamientos; y las dificultades que enfrentan las mujeres diagnosticadas con cáncer de mama, en la voz de las enfermeras. Consideraciones finales: se percibe la importancia de suscitar la reflexión de las enfermeras sobre el cuidado de la mujer con cáncer de mama, para (re) pensar la asistencia ofrecida en toda la dimensión biopsicosocio-espiritual de la mujer.

Palabras clave: Neoplasias de la mama; Atención de enfermería; Enfermería oncológica; Integralidad en salud; Investigación cualitativa. 


\section{Introdução}

O câncer de mama é o tipo mais comum entre as mulheres e uma das principais causas de mortalidade entre o sexo feminino (Inca, 2020). No mundo, segundo a Organização Mundial da Saúde, o câncer de mama representou 24,2\% do total de casos em 2018 com, aproximadamente, 2,1 milhões de novos casos diagnosticados, sendo a quinta causa de morte por câncer em geral, com 626.679 óbitos e a causa mais frequente de morte por câncer em mulheres (Inca, 2020). No Brasil, de acordo com o Ministério da Saúde, as estimativas para o ano de 2020 são de 66.280 casos novos, representando uma taxa de incidência de 43,74 casos por 100.000 mulheres (Inca, 2019).

Possivelmente, o câncer de mama é a neoplasia mais temida pelas mulheres. Compreende-se que o adoecimento pelo câncer constitui uma experiência que remete ao sofrimento e enfrentamento de múltiplas questões de ordem existencial, dado os significados distintos que implicam na vida da mulher (Birk et al., 2019). Associado a isso, a doença adquire um contorno especial pelo estigma que carrega, sendo o momento da sua descoberta vivenciado com intensa angústia, incertezas, conflitos e ansiedade, estando todo esse sofrimento associado ao caráter incurável e à ideia de possível morte (Ziguer, Bortoli \& Prates, 2016).

Cuidar dessas mulheres exige uma atitude profissional diferenciada, pela gravidade da doença e pela influência que ela exerce no aspecto biopsicossocial da paciente, sendo que, muitas vezes, desestrutura sua rotina, seus hábitos e sua vivência na sociedade (Lopes et al., 2015). Neste contexto, o enfermeiro desenvolve um papel de destaque na equipe multiprofissional, por estabelecer uma assistência de Enfermagem que proporciona cuidados que atendem às expectativas e necessidades dessas mulheres durante todos os estágios da doença. Esses cuidados recaem na oferta de conforto físico e emocional à paciente e sua família, permitindo a todos verbalizar seus sentimentos e valorizá-los, além de identificar áreas potencialmente problemáticas, auxiliar e mobilizar suas redes de apoio (Ferrari et al., 2018).

Contudo, é essencial dispor de sensibilidade para compreender e intervir frente às dificuldades as quais a mulher está passando, com uma visão humanística e integral do cuidado, preocupando-se com o estado físico, emocional, social e familiar da paciente. Faz-se importante que seu acompanhamento seja promovido com dedicação e confiança, possibilitando o restabelecimento da saúde em seu sentido mais amplo (Galdino et al., 2017).

A partir destas considerações, o presente artigo deriva de um trabalho de conclusão de curso, que teve como questão de pesquisa "Qual a percepção de cuidado e práticas desenvolvidas junto às mulheres diagnosticadas com câncer de mama na ótica de enfermeiros atuantes em um hospital de referência localizado no extremo oeste do estado de Santa Catarina?", a qual direcionou ao objetivo que foi conhecer a percepção de enfermeiros sobre o cuidado e as práticas desenvolvidas junto às mulheres com diagnóstico de câncer de mama.

A partir desta problematização, tem-se o intuito de repensar o cuidado de Enfermagem e saúde ofertados a essas mulheres, a fim de propor mudanças à qualidade de futuras políticas públicas e estratégias em saúde, com vistas a assistência de Enfermagem integral e humanizada.

\section{Metodologia}

Trata-se de uma pesquisa de delineamento qualitativo, do tipo exploratória-descritiva. A pesquisa qualitativa é a que se aplica ao estudo da história, das relações, das representações e das percepções, apresentando melhor conformação a investigações de grupos e segmentos delimitados e focalizados, de histórias sociais sob a ótica dos atores, de relações e para análises de discursos e de documentos (Minayo, 2014). Já a pesquisa descritiva-exploratória tem como objetivo a descrição de características de determinada população ou fenômeno, buscando uma maior familiaridade com o objeto do estudo, visando o esclarecimento ou a constituição de hipóteses (Gil, 2010). 
Foi realizada uma pesquisa de campo com os enfermeiros atuantes em uma unidade de internação de um hospital de referência localizado na região do extremo oeste catarinense. Estes enfermeiros atuam diretamente no cuidado de pacientes oncológicos. Dentre os critérios de inclusão foram considerados: a) ser enfermeiro graduado; b) tempo de experiência na área maior que seis meses. No que tange aos critérios de exclusão, foram excluídos os enfermeiros que se encontravam em afastamento temporário de qualquer natureza.

A coleta de dados aconteceu em outubro de 2016, por meio de entrevista semiestruturada, com perguntas abertas acerca do cuidado ofertado às mulheres com diagnóstico de câncer de mama, oportunizando aos participantes discorrer livremente sobre a temática proposta. O instrumento de coleta de dados ainda contava com perguntas fechadas, a fim de apresentar uma breve caracterização dos participantes, com relação à faixa etária, formação e atuação profissional. As entrevistas tiveram caráter individual, sendo realizadas em um espaço que garantiu a privacidade do participante. A gravação ocorreu por meio de aparelho digital com o consentimento do participante, de modo a registrar integralmente a fala, assegurando material autêntico para a análise.

Após a realização das entrevistas, ocorreu a transcrição dos dados obtidos por meio das gravações das falas das participantes de forma literal em um editor de textos, constituindo o corpus da pesquisa. O pesquisador, além de se apropriar dos dados apresentados, deve julgá-los, interpretá-los e tomar posição em relação a eles. Tanto o sujeito que comunica como aquele que o interpreta são marcados pela história, pelo tempo e pelo seu grupo (Minayo, 2014).

Os dados foram analisados por meio da análise de conteúdo do tipo temática, proposta por Minayo. Esta corresponde a um conjunto de técnicas de pesquisa que permitem inferir dados de um determinado contexto, mediante a organização, leitura e discussão dos dados coletados, partindo de uma leitura de primeiro plano dos documentos para atingir um nível mais profundo, ultrapassando os sentidos manifestos do material (Minayo, 2014).

A análise temática sucedeu em três etapas: 1) organização dos dados: identificação do material coletado, leitura inicial e primeiras impressões dos dados obtidos; 2) classificação dos dados: a partir das questões teoricamente elaboradas, foi feita seleção dos trechos mais relevantes e ideias centrais, que foram agrupados em categorias empíricas. Para este agrupamento, foi considerada a relevância da fala acerca do objeto, recorrência do tema e expressividade; 3) análise e interpretação final: movimento entre os conteúdos teóricos e empíricos, correlacionando-os, em um movimento dialético.

Para preservar o sigilo e anonimato, cada um dos dez participantes foi identificado pelo código alfanumérico como E1 (Entrevistado 1), sucessivamente, até E10 (Entrevistado 10), obedecendo a ordem em que as entrevistas foram realizadas.

Esta pesquisa seguiu criteriosamente as orientações da Resolução n ${ }^{\circ} 466$, de 12 de dezembro de 2012, do Conselho Nacional de Saúde, que dispõe sobre as diretrizes e normas regulamentadoras da pesquisa envolvendo a participação de seres humanos (Ministério da Saúde, 2012), sendo aprovada pelo Comitê de Ética e Pesquisa da Universidade do Oeste de Santa Catarina, sob o número CAAE: 57539816.5.0000.5367 e Parecer número 1.647.491.

\section{Resultados e Discussão}

Fizeram parte do estudo sete enfermeiros. Entre estes, seis são do sexo feminino e um do sexo masculino, com idade entre 24 e 38 anos. O tempo de formação variou entre um ano e meio a oito anos. Dos entrevistados, três possuem especialização na área de oncologia, um está com a especialização na área em andamento e três não possuem especialização na área.

A partir da análise qualitativa do conteúdo foi possível identificar os discursos de acordo com os aspectos abordados sobre as percepções dos enfermeiros frente ao cuidado às mulheres diagnosticadas com câncer de mama. Como resultado, emergiram dois temas: 1) Cuidados de Enfermagem às mulheres com câncer de mama: práticas e enfrentamentos; e, 2) Dificuldades enfrentadas pelas mulheres com câncer de mama, na voz dos enfermeiros. 


\section{Cuidados de Enfermagem às mulheres com câncer de mama: práticas e enfrentamentos}

Ao serem questionados sobre os cuidados ofertados às mulheres que recebem o diagnóstico de câncer de mama, percebe-se a importância que os entrevistados delegam à necessidade de uma abordagem da doença não apenas na sua condição clínica, que acomete o âmbito biológico, mas que atinge a vida da mulher na perspectiva psicológica e social do ser. Neste contexto, o apoio psicológico aparece como um dos principais cuidados destinados a essas mulheres, como forma de fortalecer a integralidade da assistência prestada.

A minha percepção é que essas mulheres precisam ter um atendimento um pouco diferenciado. Elas sofrem um abalo psicológico $(E 1)$.

[...] Eu acho que as neoplasias em si exigem uma atenção biopsicossocial nas pacientes. É necessário que a gente assista elas integralmente, como um todo, porque acredito que afeta a parte física e a parte emocional (E3).

[...] são pacientes bem mais sensíveis, tanto a parte psicológica quanto a parte fisiológica. A gente sempre tem que tratar ela como um ser integral [...] (E7).

Ao cuidar de pacientes diagnosticadas com câncer de mama, o enfermeiro deve estar preparado para atendê-las integralmente, considerando ser uma enfermidade agressiva em diversos aspectos, não se limitando a dimensão física, mas que perpassa todo o círculo que rege a vida da mulher. Para tanto, estratégias de apoio psicológico podem ajudar as pacientes a lidarem com o câncer e os efeitos dos tratamentos. Para prover cuidado integral às pacientes, é preciso conhecer suas necessidades e de que modo pode-se minimizar o impacto psicossocial da doença e os efeitos adversos de seus tratamentos (Santos \& Souza, 2019). As condutas humanizadas dos enfermeiros acabam por aliviar o peso do diagnóstico e do tratamento, contribuindo para uma melhor reabilitação da paciente, que aos poucos vai reconstruído e ressignificando sua vida.

Além do apoio ofertado às pacientes, faz-se necessário que esse cuidado se estenda também às suas famílias, que juntos vivenciam todo o processo que vai desde o diagnóstico ao tratamento da doença. A família necessita de apoio e uma atenção especial pois, na maioria das vezes, é o principal suporte desta paciente.

[...] Eu acho que o apoio psicológico para a paciente e para os familiares dela também. (E2)

[...] acho que abala muito a questão psicológica, para a paciente e para os familiares também. (E6)

\section{[...] a família também sofre com isso, não é só a paciente. (E7)}

A rede de apoio constituída por familiares, amigos e profissionais é essencial no enfrentamento da doença e na reabilitação da mulher. O apoio social provoca efeito direto na melhoria dos aspectos psicoemocionais desencadeados no processo saúde-doença. A relação entre apoio social e câncer de mama surge como uma importante estratégia de adaptação à doença, pois a rede de apoio é considerada um fator protetor e recuperador da saúde da mulher com câncer, já que a auxilia a lidar com as diferentes fases do processo de enfrentamento da doença (Vargas et al., 2020).

Para isso, o enfermeiro que cuida deve ampliar seu campo de atuação, contemplando o contexto social em que ela está inserida (Kuhn et al., 2018), ofertando-lhes suporte, valorizando e respeitando seus sentimentos e expectativas após o 
diagnóstico da doença. Entende-se que, na medida em que a mulher e seus familiares encontram-se acolhidos e amparados, apresentam melhores condições para vivenciar este período (Ziguer, Bortoli \& Prates, 2016).

Ao tomar conhecimento sobre a existência da doença, os entrevistados ressaltam a importância das orientações e esclarecimentos das dúvidas que virão, para que o medo do desconhecido seja minimizado, estando a mulher mais preparada física e psicologicamente, com vistas a um melhor enfrentamento da doença, maior adesão ao tratamento e melhores resultados do mesmo.

Eu acho que elas têm que ter um preparo para o enfrentamento da doença [...] conhecimento do que é a doença, para depois iniciar a terapia medicamentosa [...]. (E4)

\section{[...] deixar bem claro a possibilidade de tratamento, de cura, o que ela pode encontrar [...]. (E5)}

Durante o tratamento orientar algumas reações ocorridas [...] orientar que vai ter a queda do cabelo, tentar elevar a autoestima dela, para que ela não fique totalmente abalada pelas ocorrências devido ao câncer de mama. (E6)

A disponibilidade de informações sobre o tratamento e orientações quanto ao manejo de seus eventos adversos é muito importante para o gerenciamento adequado da situação, além do suporte emocional que deve ser prestado e o estímulo ao autocuidado (Gozzo et al., 2015). A prática dos enfermeiros em orientar e desenvolver ações de educação em saúde adquire relevância durante todo o processo do câncer de mama, proporcionando maior segurança e, consequentemente, uma melhor adesão ao tratamento (Ferrari et al., 2018).

Além do cuidado ofertado pelo enfermeiro, ao considerar a assistência sob uma ótica biopsicossocial e espiritual, torna-se fundamental a atuação de uma equipe multiprofissional, conforme é destacado no depoimento a seguir.

[...] a gente sempre tem o apoio da equipe multidisciplinar. Se a gente percebe que uma paciente está mais depressiva, a gente vai chamar o suporte da assistente social, psicóloga [...] ou se ela tem consulta com o médico oncologista. (E7)

No cuidado à mulher com câncer de mama, a equipe multiprofissional deve resgatar os valores humanos em seu processo de trabalho, desconstruindo o cuidado mecanizado e rotinizado, proporcionado uma práxis inovadora e transformadora da relação entre profissional e paciente, permitindo a esta mulher um atendimento holístico, promovendo o restabelecimento da saúde no âmbito biopsicossocial e espiritual (Nascimento et al., 2014).

Durante os cuidados de Enfermagem às mulheres com câncer de mama, os enfermeiros acabam se deparando com alguns enfrentamentos, considerando que os sentimentos de dor e sofrimento exteriorizados por elas também afetam os profissionais de saúde responsáveis pelo seu cuidado.

[...] Eu acho que essa condição de estar desenvolvendo um câncer afeta bastante o psicológico dos pacientes e da equipe como um todo [...]. (E3)

Os profissionais que assistem estas pacientes também precisam ser acompanhados, para que a rotina do cuidado não se torne desgastante e a dor do outro não seja absorvida de uma forma que, ao invés de cuidar, necessitam serem cuidados. Trata-se de um ser humano cuidando da dor de outro ser humano, que pode associar suas próprias vivências de entes queridos 
que adoeceram ou, até mesmo, perdidos por esta doença. As atitudes protetoras para com o paciente podem acabar trazendo consequências que podem interferir na qualidade da relação entre ambos, causando também sofrimento ao profissional (Geovanini \& Braz, 2013).

O despreparo de muitos profissionais também se coloca como uma dificuldade no cuidado à paciente, principalmente na abordagem inicial após a descoberta do diagnóstico.

Eu acho que é a abordagem inicial, a notícia. Acho que isso impacta muito, desespera muito [...]. (E4)

\section{[...] falta um pouco como chegar, como abordar essa paciente, como vou tratar ela, como eu vou conversar com ela [...]. (E6)}

A emoção, inerente ao ser humano, está presente em todos os aspectos da vida e fica mais evidente no processo de cuidar. Para os enfermeiros, conviver com o sofrimento diário dos pacientes é um grande desafio. Aliado a isso, existe o despreparo em lidar com este tipo de situação, podendo levá-lo a assumir atitudes extremas. Por um lado, o profissional pode assumir uma postura distante e evitativa, na qual o profissional apenas trata da porção biológica da paciente. Por outro, o enfermeiro pode adquirir uma atitude participante e comprometida, quando existe a proximidade excessiva e invasiva, com a supervalorização do lado emocional (Menegócio, Rodrigues \& Teixeira, 2015).

Entretanto, o relacionamento do enfermeiro com o paciente nunca é neutro. O enfermeiro deve reconhecer que sua presença é tão importante quanto o procedimento técnico. A prestação destes cuidados é considerada efetiva quando o enfermeiro tem propriedade sobre a patologia e habilidade em lidar com os sentimentos dos outros e com as próprias emoções frente ao doente, com ou sem possibilidade de cura (Sousa et al., 2009).

Cabe destacar que, em alguns depoimentos, são relatadas situações de maior dificuldade na oferta de cuidados à paciente que se encontra em estágio mais avançado da doença, sem possibilidades de cura.

Eu acredito que é lidar com a paciente que está em fase terminal [...]. (E1)

\section{[...] aquelas em fase terminal [...]. (E2)}

[...] eu acho que lidar com a terminalidade. Quando a paciente já tem um diagnóstico de tratamento paliativo, eu acho que dificulta bastante. (E3)

O sentimento de finitude e a morte sendo encarada como inaceitável para muitos, é um dos fatores que contribuem para o despreparo dos enfermeiros para compreender o processo de morte e o morrer. Os enfermeiros devem ter em mente que a essência do cuidar envolve propiciar a melhor qualidade de vida, no tempo de vida do paciente sem perspectiva de cura (Menegócio, Rodrigues \& Teixeira, 2015). Apesar de inevitável, a morte não é uma questão simples de ser discutida, uma vez que, em nossa cultura, é representada pelo pavor e pela não aceitação. É neste cenário de diversidade com relação à morte que se encontram os enfermeiros, vivendo em constante desafio, uma vez que diariamente permanecem em conflito, lutando pela vida e contra a morte, tomando para si a responsabilidade de salvar, curar ou aliviar, procurando sempre preservar a vida, já que a morte, na maioria das vezes, é vista por estes profissionais como um fracasso, sendo, desta forma, duramente combatida (Sousa et al., 2009). 


\section{Dificuldades enfrentadas pelas mulheres com câncer de mama, na voz dos enfermeiros}

O primeiro desafio a ser enfrentado pela mulher é o momento do diagnóstico. Para o enfermeiro, a descoberta do diagnóstico e a aceitação do 'estar doente' é muito difícil para a paciente, por desacreditar na sua cura. A mulher se depara com momentos de conflitos, incertezas e questionamentos: ‘- Por que comigo?'. Emerge o desejo de saber o porquê foram 'escolhidas' para viver esta enfermidade.

[...] acredito também essa parte de aceitação (do diagnóstico) [...] Ainda hoje, o câncer, quando diagnosticado, é tido como uma sentença de morte. (E3)

Desde a descoberta do diagnóstico do câncer de mama, as mulheres vivenciam sentimentos negativos, como ansiedade, medo, depressão, angústia e desespero; em que muitas passam pelo chamado processo de negação. Aquelas que vivem este processo, geralmente na fase inicial da doença, negam a enfermidade, levando-as, muitas vezes, à recusa do fato de estarem doentes. Ao descobrir-se com câncer, a mulher inicia uma nova trajetória em sua vida, que vai desde a aceitação da doença até a sua readaptação e ajustamentos psicossociais, devido a condição de vulnerabilidade e de perdas emocionais ocasionadas pela doença. Esse fato traz mudanças significativas no cotidiano da mulher, cuja incerteza quanto ao futuro somam-se ao desespero e ao medo de morrer (Vargas et al., 2020).

A mastectomia surge como outro enfrentamento decorrente do câncer de mama. É uma das escolhas terapêuticas mais utilizadas, representando forte ameaça à mulher, sendo vista como a mutilação de um órgão que representa sua feminilidade, com consequências profundas em diversos sentidos, como desconforto físico e psicológico, depressão, mudanças na autoimagem e baixa autoestima.

[...] passar por aquele processo quanto à mutilação, a mastectomia [...] são partes do corpo que são mutiladas [...]. (E1)

\section{[...] vai ter que retirar a mama, como que vai ser a aceitação da família e a tua própria aceitação [...]. (E6)}

Por que o câncer de mama é como se fosse uma amputação traumática, a amputação da mama. (E7)

Para a mulher a mama representa um símbolo de feminilidade, sendo que sua imagem corporal contribui de forma direta para sua autoestima. Após a mastectomia, muitas mulheres têm dificuldade de escolher uma roupa para vestir, ir à praia ou ter um contato físico com outra pessoa por conta da alteração corporal sofrida (Silva, Arboit \& Menezes, 2020). Com a perda da mama, a mulher vivencia um conjunto de sentimentos negativos que influenciam no seu bem-estar físico e emocional, os quais ferem a sexualidade e a identidade feminina ao trazer prejuízos significativos para a autoestima e autovalorização (Rocha et al., 2019).

Associada à mastectomia, em alguns casos, a mulher se depara com a perda do cabelo, outro fator determinante para a imagem corporal da mulher e sua representatividade feminina.

\section{[...] com o tratamento vem a queda de cabelo e isso para a mulher é uma coisa difícil de enfrentar [...]. (E7)}

A mastectomia causa grande impacto na vida da mulher e, quando associada à quimioterapia, ele aumenta em função dos efeitos colaterais associados aos antineoplásicos, como a perda de seus cabelos, sobrancelhas e cílios. A perda do cabelo, 
além de afetar a imagem corporal, retrata o sofrimento da doença, trazendo à memória as vivências do adoecimento diante das marcas deixadas em seus corpos. Essas repercussões ultrapassam os aspectos relacionados a autoimagem e influenciam as relações interpessoais e sociais da sua vida (Reis \& Gradim, 2018).

No próximo depoimento, percebe-se que, além da alteração da imagem corporal, a mulher tem de lidar com os efeitos adversos ocasionados pelo tratamento quimioterápico.

\section{[...] efeitos dos quimioterápicos, vômito, indisposição [...]. (E3)}

Apesar dos avanços conquistados, ainda hoje a quimioterapia causa muitos efeitos colaterais. Ela é considerada a principal fonte de dor e sofrimento causados pelas reações dos medicamentos e pela perda ou diminuição da autoestima provocada por eles. Queda dos cabelos, falta de energia, fadiga, perda de peso, náuseas e vômitos são sintomas frequentemente relatados por pacientes que passam pelo tratamento e têm sua vida social também afetada pelos seus efeitos (Ferrari et al., 2018).

A acessibilidade também se traduz como uma dificuldade que as mulheres com câncer de mama enfrentam. Principalmente em municípios menores, os quais não dispõem de tratamento oncológico, há a necessidade de as pacientes se deslocar a outros municípios que disponibilizem o tratamento por meio do Sistema Único de Saúde.

\section{[...] o tratamento geralmente é longe e elas têm que passar na estrada para fazer quimioterapia. (E2)}

[...] acho que é a dificuldade no acesso, porque ainda não somos referência, estamos implantando a referência. Então, as pacientes têm que se deslocar até (outro município) para fazer o tratamento [...]. (E3)

O tratamento a que muitas mulheres são submetidas é bastante agressivo, deixando-as debilitadas, o que requer maiores cuidados para que haja uma melhor resposta na reabilitação desta paciente, tornando-se mais desafiador quando este não é ofertado no município em que a paciente reside, tendo que lidar com o deslocamento de uma cidade à outra, o que gera desgaste físico e estresse. Ainda, aquelas que não possuem condições de se deslocar com carros próprios e dependem de carros da saúde disponibilizados pelos municípios de origem, estão expostas ao contato direto com outros pacientes de diversas patologias e necessitam esperar a realização dos procedimentos de todos os pacientes para o retorno à sua cidade, ficando expostas a um alto risco de desestabilização e possíveis contaminações (Ferrari et al., 2018).

\section{Considerações Finais}

Por meio do desenvolvimento e resultados oriundos desta pesquisa, foi possível analisar diligentemente a percepção dos enfermeiros frente aos cuidados às mulheres diagnosticadas com câncer de mama. Assim, fica evidenciada a importância do cuidado à mulher em sua totalidade. Para tanto, a relação estabelecida entre enfermeiro-paciente-família torna-se fundamental para a exteriorização dos sentimentos provocados pela doença, considerando que cada ser é único e experencia este processo de forma particular. Este olhar ampliado para o cuidado auxilia a mulher na superação dos obstáculos impostos pelo diagnóstico de uma doença carregada de estigmas, além de contribuir para os próprios enfrentamentos dos enfermeiros que cuidam e não estão neutros neste processo.

Com base nos resultados desta pesquisa, considera-se que esta poderá auxiliar os profissionais de saúde, em particular da Enfermagem, a legitimar ou revisar suas práticas de cuidado relativas à mulher que enfrenta o câncer de mama, considerando que o apoio ofertado é importante para a aceitação e reestruturação na vida da mulher. Para o público em geral, a 
pesquisa traz um exemplo atualizado do conhecimento produzido e disponibilizado para a comunidade acadêmica a respeito da temática, representando uma importante contribuição e indicativo para estudos futuros.

\section{Referências}

Birk, N. M., Girardon-Perlini, N. M. O., Lacerda, M. R., Terra, M. G., Beuter, M., \& Martins, F. C. (2019). Percepção de mulheres com câncer de mama sobre o cuidado de enfermagem à espiritualidade. Cienc Cuid Saude, 18(1), e45504. http://periodicos.uem.br/ojs/index.php/CiencC uidSaude/article/view/45504/751375139227

Ferrari, C. F., Abreu, E. C., Trigueiro, T. H., Silva, M. B. G. M., Kochla, K. A., \& Souza, S. R. R. K. (2018). Orientações de cuidado do enfermeiro para a mulher em tratamento para câncer de mama. Rev Enferm UFPE Online, 12(3), 676-683. https://pdfs.semanticscholar.org/2930/57ca3f5840f8e1 3a5c97e17e6771c9c2431b.pdf

Galdino, A. R., Pereira, L. D. A., Neto, S. B. C., Souza, C. B., \& Amorim, M. H. C. (2017). Qualidade de vida de mulheres mas tectomizadas matriculadas em um programa de reabilitação. Rev Fund Care Online, 9(2), 451-458. http://www.seer.unirio.br/index.php/cuidadofundamental/article/view/5440/pdf_1

Geovanini, F., \& Braz, M. (2013). Conflitos éticos na comunicação de más notícias em oncologia. Rev Bioét, 21(3), 455-462. https://www.scielo.br/pdf/bioet/v21n3/a10v21n3.pdf

Gil, A. C. (2010). Como elaborar projetos de pesquisa. Atlas.

Gozzo, T. O., Souza, S. G., Moysés, A. M. B., Carvalho, R. A. O., \& Ferreira, S. M. A. (2015). Conhecimento da equipe de Enfermagem acerca de eventos adversos do tratamento quimioterápico. Cienc Cuid Saúde, 14(2), 1058-1066. http://periodicos.uem.br/ojs/index.php/CiencCuidSaud e/article/view/25040/pdf_363

Instituto Nacional de Câncer José Alencar Gomes da Silva. (2019). Estimativa 2020: incidência do câncer no Brasil. INCA. https://www.inca.gov.br/publicacoes/livros/estimativa-2020-incidencia-de-cancer-no-brasil

Instituto Nacional de Câncer José Alencar Gomes da Silva. (2020). Câncer de mama. INCA. https://www.inca.gov.br/tipos-de-cancer/cancer-de-mama

Instituto Nacional de Câncer José Alencar Gomes da Silva. (2020). Conceito e magnitude do câncer de mama. INCA. https://www.inca.gov.br/controledo-cancer-de-mama/conceito-e-magnitude

Kuhn, C. I., Simionato, D. G. S., Bortoli, C. F. C., \& Coelho, J. B. A. (2018). Casa de apoio: suporte às mulheres com câncer de mama. J. nurs. Health, 8(3), e188310. https://periodicos.ufpel.edu.br/ojs2/index.php/enfermagem/article/view/14507/9062

Lopes, M., Silva, A. C., Ferreira, A. M., \& Lino, A. A. C. F. (2015). Revisão narrativa sobre a humanização da assistência pela equipe de Enfermagem na área oncológica. Rev Eletron. Gestão \& Saúde, 6(3), 2373-2390. https://periodicos.unb.br/index.php/rgs/article/view/3115/2800

Menegócio, A. M., Rodrigues, L., \& Teixeira, G. L. (2015). Enfermagem oncologia: relação de afetividade ou meramente técnica? Ensaios Cienc., Cienc. Biol. Agrar. Saude, 19(3), 118-123. https://www.redalyc.org/pdf/260/26042169004.pdf

Minayo, M. C. S. (2014). O desafio do conhecimento: pesquisa qualitativa em saúde. (14a ed.), Hucitec.

Ministério da Saúde (BR). (2012). Resolução $n^{o} 466$, de 12 de dezembro de 2012 . Conselho Nacional de Saúde. Brasília. http://conselho.saude.gov.br/resolucoes/2012/Reso466.pdf

Nascimento, K. T. S., Fonsêca, L. C. T., Andrade, S. S. C., Leite, K. N. S., Zaccara, A. A. L., \& Costa, S. F. G. (2014). Cuidar integral da equipe multiprofissional: discurso de mulheres em pré-operatório de mastectomia. Esc Anna Nery, 18(3), 435-440. https://www.scielo.br/pdf/ean/v18n3/1414-8145ean-18-03-0435.pdf

Reis, A. P. A., \& Gradim, C. V. C. (2018). A alopécia no câncer de mama. Rev Enferm UFPE Online, 12(2), 447-455. https://periodicos.ufpe.br/revistas/revistaenfermagem/article/viewFile/25097/27856

Rocha, C. B., Fontenele, G. M. C., Macêdo, M. S., Carvalho, C. M. S., Fernandes, M. A., Veras, J. M. M. F., \& Silva, J. S. (2019). Sentimentos de mulheres submetidas à mastectomia total. Rev Cuid, 10(1), e606. https://revistacuidarte.udes.edu.co/index.php/cuidarte/article/view/606/1075

Santos, M. A., \& Souza, C. (2019). Intervenções grupais para mulheres com câncer de mama: desafios e possibilidades. Psic.: Teor. e Pesq., 35, e35410. https://www.scielo.br/pdf/ptp/v35/1806-3446-ptp-35-e35410.pdf

Silva, F. C. N., Arboit, E. L., \& Menezes, L. P. (2020). Enfrentamento de mulheres diante do tratamento oncológico e da mastectomia como repercussão do câncer de mama. Rev Fun Care Online, 12, 357-63. http://seer.unirio.br/index.php/cuidadofundamental/article/viewFile/7136/pdf_1

Sousa, D. M., Soares, E. O., Costa, K. M. S., Pacífico, A. L. C., \& Parente, A. C. M. (2009). A vivência da enfermeira no processo de morte e morrer dos pacientes oncológicos. Texto Contexto - Enferm, 18(1), 41-47. https://www.scielo.br/pdf/tce/v18n1/v18n1a05.pdf

Vargas, G. S., Ferreira, C. L. L., Vacht, C. L., Dornelles, C. L., Silveira, V. N., \& Pereira, A. D. (2020). Rede de apoio social à mulher com câncer de mama. Rev Fun Care Online, 12, 73-78. http://seer.unirio.br/index.php/cuidadofundamental/article/view/7030/pdf

Ziguer, M. L. P. S., Bortoli, C. F. C., \& Prates, L. A. (2016). Sentimentos e expectativas de mulheres após diagnóstico de câncer de mama. Espaço para a Saúde - Revista de Saúde Pública do Paraná, 17(1), 108-113. http://espacoparasaude.fpp.edu.br/index.php/espacosaude/article/view/374/14 